\title{
Correction to: New constructions of entanglement-assisted quantum codes
}

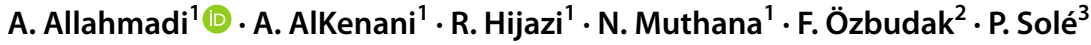

Published online: 6 October 2021

๑) Springer Science+Business Media, LLC, part of Springer Nature 2021

\section{Correction to: Cryptography and Communications https://doi.org/10.1007/s12095-021-00499-7}

The original version of this article unfortunately missed to include another Acknowledgments below.

"The authors acknowledge the financial support provided by the NSTIP strategic technologies program in the Kingdom of Saudi Arabia-Project No (12-MAT305503), and extend the thanks to the Science and Technology Unit, King Abdulaziz University for their technical support."

The original paper was updated.

Publisher's note Springer Nature remains neutral with regard to jurisdictional claims in published maps and institutional affiliations.

The original article can be found online at https://doi.org/10.1007/s12095-021-00499-7.

A. Allahmadi

adelnife2@yahoo.com

A. AlKenani

aalkenani10@hotmail.com

R. Hijazi

rhijazi@kau.edu.sa

N. Muthana

najat_muthana@hotmail.com

F. Özbudak

ozbudak@metu.edu.tr

P. Solé

sole@enst.fr

1 Mathematics Department, King Abdulaziz University, Jeddah, Saudi Arabia

2 Department of Mathematics and Institute of Applied Mathematics, Middle East Technical University, Ankara, Turkey

3 I2M, Aix Marseille University, Centrale Marseille, CNRS, Marseille, France 